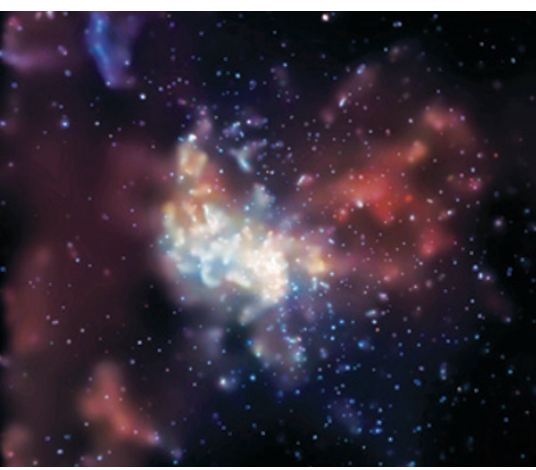

ASTRONOMY

\section{Reflections from a black hole}

Sagittarius $A^{*}$, the unusually dim supermassive black hole at the heart of the Milky Way

(pictured), may have flared up at times during the past few centuries.

Observations from NASA's Chandra X-ray Observatory between 1999 and 2011 reveal bright patches of X-rays in molecular clouds in the centre of the Galaxy. The glow, which has been rippling outwards, could be reflected radiation that was emitted from the black hole long ago, and then bounced off nearby clouds.

Maica Clavel of the Paris Diderot University and her colleagues calculate that two sudden surges in the black hole's activity - one lasting no more than two years, and the other about a decade could produce the observed reflections. Charting them may help to reveal what the black hole has been consuming. Astron. Astrophys. 558, A32 (2013)

\section{CANCER}

\section{Two-faced cancer gene}

A genetic variant that greatly boosts the risk of testicular cancer may protect lightskinned individuals from skin cancer by helping them to tan.

A team led by Douglas Bell at the US National Institutes of Health in Bethesda, Maryland, and Gareth Bond at the University of Oxford, $\mathrm{UK}$, surveyed data from genome-wide association studies (GWAS). They focused on polymorphisms in DNA binding sites for the tumoursuppressor protein $\mathrm{p} 53$. One variant, in a gene called KITLG, has one of the strongest effects of any pro-cancer variant identified by GWAS and was vastly more common in caucasians. In mice, the p53-KITLG interaction boosted the growth of pigmentproducing cells after exposure to ultraviolet radiation, and so might protect against excessive sun damage and cancer. Cell 155, 410-422 (2013)

\section{CLIMATE SCIENCE}

\section{Ozone hole fans African heat}

Much of the summer warming in southern Africa over recent decades seems to have been due to the ozone hole over Antarctica.

Desmond Manatsa, of Bindura University of Science in Zimbabwe, and colleagues compared regional climates before and after ozone depletion set in around 1993. Pronounced surface warming strongly correlated with shifts in the strength and position of pressure systems in the atmosphere that enhance the southward flow of warm tropical air. These shifts are often attributed to ozone depletion in the upper atmosphere.

The expected closure of the ozone hole by 2050 may help to mitigate climate warming in southern Africa, the authors conclude.

Nature Geosci.

http://doi.org/n8x (2013)

For a longer story on this research, see go.nature.com/ph2nyo

How exercise
boosts the brain

A protein molecule secreted by muscles during exercise boosts the expression of factors that help to protect brain neurons.

Endurance exercise is known to induce production

COMMUNITY CHOICE

The most viewed papers in science

\title{
SUSTAINABILITY
}

\section{Steel mesh sucks in fog}

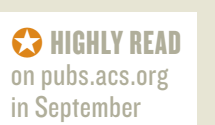

One square metre of high-tech mesh could capture 12 litres of potable water a day from morning fog.

Fog-harvesters are used in countries such as Chile to collect drinking water. Tiny fog droplets in humid air blow through mesh filaments, where they coalesce into larger drops that roll into a collecting trough.

To boost the amount of water captured, Robert Cohen and Gareth McKinley at the Massachusetts Institute of Technology in Cambridge and their colleagues systematically modelled and tested surface chemistries, fibre thicknesses and geometrical configurations of woven mesh. They produced a mesh of thin steel strands coated with a fluorinated polymer. In laboratory tests simulating Chilean mountain fog, the mesh collected water at rates fivefold higher than conventional fog-catching meshes and came close to the theoretical limit calculated by the researchers.

Langmuir http://doi.org/n7n (2013)

of a protein called irisin and to improve cognitive performance in patients with some neurological conditions, but the mechanism linking these observations has been unclear.

A team led by Michael Greenberg and Bruce Spiegelman at Harvard Medical School in Boston, Massachusetts, observed that irisin levels increased in a brain area associated with learning and memory after mice ran on exercise wheels. When levels of irisin or its progenitor were raised experimentally in the blood and in neurons, genes associated with learning and memory became active in the brain.

Cell Metab. http://doi.org/n8z (2013)

$$
\text { BIOMECHANICS }
$$

\section{Jellyfish wake makes power}

When a jellyfish swims, its pulsing body gets an extra push from spinning water in its wake (pictured, red).

Brad Gemmell at the Marine Biological Laboratory in Woods Hole, Massachusetts, and his colleagues studied how water flows around pulsing or paralysed moon jellyfish (Aurelia aurita). The animal's bell-shaped body produces vortex rings of water as it pulsates.

As the bell flattens and expands, one of the rings rolls inside the bell and sucks in more water. That pushes the jellyfish forward without any extra force from the creature's muscles. This makes the simple predator one of the most energy-efficient swimmers on the planet, the authors say.

Proc. Natl Acad. Sci USA http://doi.org/n7k (2013) For a longer story on this research, see go.nature.com/gbbmhv

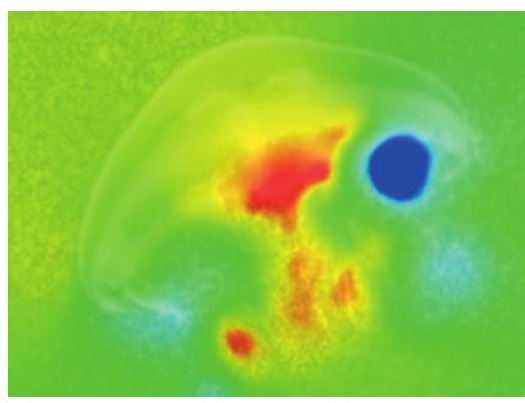

\section{$\rightarrow$ NATURE.COM}

For the latest research published by Naturevisit:

www.nature.com/latestresearch 